\title{
Partial Difference Equation Method for Lattice Path Problems
}

\author{
R. Brak ${ }^{1}$, J.W. Essam ${ }^{2}$, and A.L. Owczarek ${ }^{1}$ \\ ${ }^{1}$ Department of Mathematics and Statistics, The University of Melbourne, Parkville, \\ VIC 3052, Australia \\ brak@maths.mu.oz.au, aleks@ms.unimelb.edu.au \\ ${ }^{2}$ Department of Mathematics, Royal Holloway, University of London, Egham, \\ Surrey TW20 0EX, England \\ j.essam@vms.rhbnc.ac.uk \\ Received $* * *$
}

AMS Subject Classification: ***

\begin{abstract}
Many problems concerning lattice paths, especially on the square lattice have been accurately solved. For a single path, many methods exist that allow exact calculation regardless of whether the path inhabits a strip, a semi-infinite space or infinite space, or perhaps interacts with the walls themselves. It has been shown that a transfer matrix method using the Bethe Ansatz allows for the calculation of the partition function for many non-intersecting paths interacting with a wall. This problem can also be considered using the Gessel-Viennot methodology. In a concurrent development, two non-intersecting paths interacting with a wall have been examined in semi-infinite space using a set of partial difference equations.

Here, we review this partial difference equation method for the case of one path in a half plane. We then demonstrate that the answer for arbitrary numbers of non-intersecting paths interacting with a wall can be obtained using this method. One reason for doing this is its pedagogical value in showing its ease of use compared to the transfer matrix method. The solution is expressed in a new form as a "constant term" formula, which is readily evaluated. More importantly, it is the natural method that generalizes easily to many intersecting paths where there is inter-path interactions (e.g., osculating lattice paths). We discuss the relationship of the partial difference equation method to the transfer matrix method and their solution via a Bethe Ansatz.
\end{abstract}

Keywords: directed paths, interacting walks, lattice paths, difference equations, constant term, Gessel-Viennot theorem, Bethe Ansatz, transfer matrix method

\section{Introduction}

Recently, the problems of one- and two-directed paths interacting with one and two walls via contact interactions on the square lattice has been accurately solved [4]. In 
particular, the partition functions for fixed length and fixed starting and ending positions have been evaluated. Another recent development [3] has been the proof of two theorems concerning the evaluation of the partition function of $N$ such paths with arbitrary inhomogeneous (with respect to the direction perpendicular to the directedness) weights; these theorems give the answer in terms of a determinant provided the solution of the one-path problem can be structured in a particular fashion. In a further work [2], it was shown that the one-path solution for the case of surface contact interactions with homogeneous bulk weights satisfies the conditions of those theorems, and hence, a solution of $N$ paths in a strip and in a half-plane interacting with their surfaces was given for all numbers of paths $N$. These formulae generalize the "master formulae" of Equation (5.9) of [5] and Equation (4) of [6] where paths that do not interact with a surface are considered. This method of solution calculates the partition function for paths between two walls (in a strip) and then finds the half plane (one wall) partition function as a limit. This is based on a transfer matrix method. However, the original work on one and two paths [4] found the solution directly in the half plane via the solution of a set of partial difference equations. This had two advantages: First, the solution of the half-plane was direct, and second, the solution came out naturally as the "constant term" of a Laurent series which was simple to evaluate. In fact, the solution of the onepath problem is substantially simpler using the partial difference equation method. As has now been shown by its use in the osculating path problem, this method, because of its constant term solution, is the method that easily generalizes, not only to many paths, but also to problems outside the range of the Gessel-Viennot theorem [7,8] where paths are allowed to intersect (but not cross).

In this paper, we generalize the partial difference equation method by applying it to the case of the $N$-path problem of Brak et. al. [2] in the half plane. This demonstrates its ease of use compared to the transfer matrix method though the partial difference method currently lacks rigor. We shall see that the solution involves using an Ansatz for the trial solution of the partial difference equations that is similar to the Bethe Ansatz for the components of the eigenvectors of the transfer matrices for the problem. This leads us to discuss the relationship between the partial-difference method and the transfer matrix method.

\section{Partial Difference Method for Non-Intersecting Paths and a Wall}

\subsection{The Model}

A lattice path or walk in this paper is a path on a square lattice rotated $45^{\circ}$ which has steps in only the north-east or south-east directions. See Figure 1 where variables $y^{i}, y^{f}$ and $t$ are shown. The path closest to the lower wall has weight $v(1) w(1,2) w(2,3)$ $w(3,2) \cdots w(1,0) w(0,1)=\kappa^{3}$ so that $y_{1}^{i}=1$ and $y_{1}^{f}=1$. A set of paths is nonintersecting if they have no sites in common. First, we describe the general model considered by Brak et. al. [3] in a strip of width $L$. This requires the following subdomains of $\mathbb{Z}^{N}$ :

$$
\stackrel{o}{S}_{L}=\{y \mid 0 \leq y \leq L, y \in \mathbb{Z} \text { and } y \text { odd }\}
$$




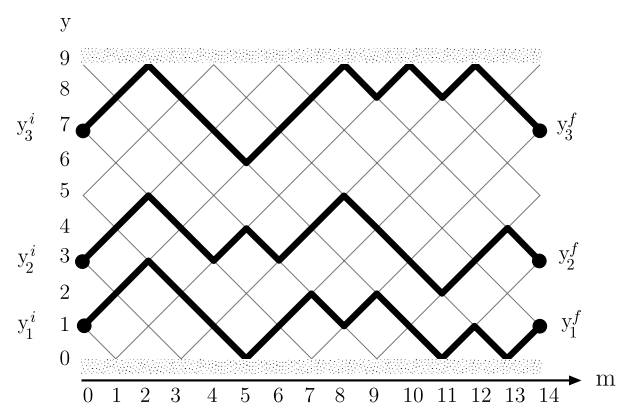

Figure 1: Three non-intersecting directed paths of length $t=14$ in a strip of width $L=9$.

$$
\begin{aligned}
& \stackrel{e}{\mathcal{S}}_{L}=\{y \mid 0 \leq y \leq L, y \in \mathbb{Z} \text { and } y \text { even }\}, \\
& \mathcal{S}_{L}=\{y \mid 0 \leq y \leq L, y \in \mathbb{Z}\}, \\
& \stackrel{o}{\mathcal{U}}_{L}=\left\{\left(y_{1}, \ldots, y_{N}\right) \mid 1 \leq y_{1}<\ldots<y_{N} \leq L, y_{i} \in \stackrel{o}{\mathcal{S}}_{L}\right\}, \\
& \stackrel{e}{\mathcal{U}}_{L}=\left\{\left(y_{1}, \ldots, y_{N}\right) \mid 0 \leq y_{1}<\ldots<y_{N} \leq L, y_{i} \in \stackrel{e}{S}_{L}\right\}, \\
& \mathcal{U}_{L}=\left\{\left(y_{1}, \ldots, y_{N}\right) \mid 0 \leq y_{1}<\ldots<y_{N} \leq L, y_{i} \in \mathcal{S}_{L}\right\} .
\end{aligned}
$$

We will use $\stackrel{p}{\mathcal{U}_{L}}$ to denote $\stackrel{o}{\mathcal{U}_{L}}$ or $\stackrel{e}{\mathcal{U}_{L}}$. Note that in most cases, it will be clear from the context whether $\stackrel{p}{\mathcal{U}_{L}}$ is $\stackrel{o}{\mathcal{U}}_{L}$ or $\stackrel{e}{\mathcal{U}}_{L}$ and thus it will not be explicitly stated unless an ambiguity arises. With the above definitions, the cardinality of the sets are

$$
\left|\stackrel{o}{\mathcal{U}}_{L}\right|=\left(\begin{array}{c}
\left\lfloor\frac{L+1}{2}\right\rfloor \\
N
\end{array}\right) \text { and }\left|\stackrel{e}{\mathcal{U}}_{L}\right|=\left(\begin{array}{c}
\left\lfloor\frac{L}{2}\right\rfloor+1 \\
N
\end{array}\right)
$$

and

$$
\left|\mathcal{U}_{L}\right|= \begin{cases}2\left(\begin{array}{c}
\left\lfloor\frac{L+1}{2}\right\rfloor \\
N
\end{array}\right) \frac{L+2-N}{L+2}, & L \text { even } \\
2\left(\begin{array}{c}
\left\lfloor\frac{L+1}{2}\right\rfloor \\
N
\end{array}\right), & L \text { odd }\end{cases}
$$

Note that this shows that the cardinalities of $\stackrel{o}{\mathcal{U}} L$ and $\stackrel{e}{\mathcal{U}}_{L}$ are the same if $L$ is odd, but they differ if $L$ is even.

Let $N$ non-intersecting paths, confined to a strip of width $L$, start at positions $\mathbf{y}^{i}=$ $\left(y_{1}^{i}, \ldots, y_{N}^{i}\right) \in \stackrel{p}{\mathcal{U}}_{L}$ in column $m=0$ of the lattice sites and terminate after $t$ steps at positions $\mathbf{y}^{f}=\left(y_{1}^{f}, \ldots, y_{N}^{f}\right) \in \mathscr{U}^{p^{\prime}}$ in the $t$ th column. If $t$ is even, then $p^{\prime}=p$, else $p^{\prime}=\bar{p}$, where $\bar{p}$ is the opposite parity to $p$. Paths are considered such that if $(m-1, y)$ 
is the position of a path in column $m-1$, the only possible positions for that path in column $m$ are $\left(m, y^{\prime}\right)$ with $y^{\prime}=y \pm 1$ provided $y, y^{\prime} \in \mathcal{S}_{L}$. Non-intersection is defined through the constraint that, if there are $N$ sites occupied at $m=0$, then in each column of sites $(0 \leq m \leq t)$, there are exactly $N$ occupied sites. The path problem associated with the five-vertex problem [9,11] was generalized by Brak et. al. [3] by the assignment of a weight $w\left(y, y^{\prime}\right)$ to the lattice edge from site $(m-1, y)$ to $\left(m, y^{\prime}\right)$ with $y^{\prime}=y \pm 1$ (see Figure 1). Note that, since $w\left(y, y^{\prime}\right)$ is assumed independent of the column index $t$, due to the square lattice structure, the weights are periodic in the $t$ direction with period two: If $y \in \stackrel{p}{S}_{L}$, then $y^{\prime} \in \mathcal{P}_{L}^{\bar{S}}$. In general, $w\left(y, y^{\prime}\right) \neq w\left(y^{\prime}, y\right)$. An arbitrary weight $v\left(y^{i}\right)$ was also associated with each of the sites occupied at $m=0$. The weight associated with a given set of paths is the product of $w$ weights over all edges occupied by the paths multiplied by the product of the $v$ weights for each of the initial sites occupied.

The partition function $\overline{\bar{Z}}_{t} \mathcal{N}\left(\mathbf{y}^{i} \rightarrow \mathbf{y}^{f}\right)$ is the sum of these weights over all sets of paths connecting $\mathbf{y}^{i}$ and $\mathbf{y}^{f}$ :

$$
\overline{\bar{Z}}_{t}^{\mathcal{N}}\left(\mathbf{y}^{i} \rightarrow \mathbf{y}^{f}\right)=\sum_{\mathcal{Y}} \prod_{j=1}^{N} v\left(y_{j}(0)\right) \prod_{m=1}^{t} w\left(y_{j}(m-1), y_{j}(m)\right)
$$

where $y_{j}(m)$ is the position of the $j$ th path in column $m$ and the set $\mathcal{Y}$ is given by

$$
\begin{aligned}
\mathcal{Y}= & \left\{y_{j}(m) \mid 1 \leq j \leq N, 0 \leq m \leq t, 1 \leq y_{1}(m)<y_{2}(m)<\cdots<y_{N}(m) \leq L\right. \\
& \left.y_{j}(m)=y_{j}(m-1) \pm 1 \text { and } y_{j}(0)=y_{j}^{i}, y_{j}(t)=y_{j}^{f}\right\}
\end{aligned}
$$

In this paper, as was discussed Brak et. al. [2], we are interested in the case when $L \rightarrow \infty$, with the weights given by

$$
w\left(y, y^{\prime}\right)= \begin{cases}\kappa, & \text { for }\left(y, y^{\prime}\right)=(1,0) \\ 1, & \text { otherwise }\end{cases}
$$

provided $y, y^{\prime} \in \mathcal{S}_{\infty}$ and

$$
v(y)= \begin{cases}\kappa, & \text { for } y=0 \\ 1, & \text { otherwise }\end{cases}
$$

provided $y \in \mathcal{S}_{\infty}$. It should be noted that while we have a model where weights are associated with edges, the model chosen is exactly equivalent to 1 , where a weight $\kappa$ is associated with every occupied site at $y=0$.

Now, we re-derive the solution of the problem described above in the half plane using a set of coupled partial difference equations. We begin by reviewing the solution of the one-path problem.

\subsection{One Path in the Half Plane}


Although this problem can be solved by many methods, for completeness, we briefly discuss the partial difference equation method as presented by Brak, Essam and Owczarek [4]. The reason for this is that the partial difference equation method is one of the very few methods that generalize to many paths. We restrict the discussion in this section to the domain $1<\kappa<2$ (see [4] for the analytical complications that arise in the extended domain $\kappa>1$ ). Note that the final generating function is a polynomial and thus, by analytic continuation, applies for all $\kappa$.

Consider a single path of length $t$ which starts at $y^{i} \in \mathcal{S}_{\infty}$ and terminates at $y^{f} \in \mathcal{S}_{\infty}$. A path of length $t$ can be made by appending a single step to a path of length $t-1$ and this consideration gives the partial difference equations

$$
\begin{aligned}
\bar{Z}_{t}^{S}\left(y^{i} \rightarrow y\right) & =\bar{Z}_{t-1}^{\mathcal{S}}\left(y^{i} \rightarrow y+1\right)+\bar{Z}_{t-1}^{\mathcal{S}}\left(y^{i} \rightarrow y-1\right), \quad y>0, \quad t>0, \\
\bar{Z}_{t}^{\mathcal{S}}\left(y^{i} \rightarrow 0\right) & =\kappa \bar{Z}_{t-1}^{\mathcal{S}}\left(y^{i} \rightarrow 1\right), \quad y=0, \quad t>0, \\
\bar{Z}_{0}^{\mathcal{S}}\left(y^{i} \rightarrow y^{f}\right) & =\left(1+(\kappa-1) \delta_{0, y^{i}}\right) \delta_{y^{i}, y^{f}},
\end{aligned}
$$

where $\delta_{x, y}$ is the Kronecker delta. The first equation (2.8) is a "bulk" equation valid for $y>0$, while (2.9) is clearly a boundary condition and (2.10) is an initial condition. To solve (2.8), we begin by separating the variables with a trial solution of $\bar{Z}_{t}^{S}\left(y^{i} \rightarrow y\right)=$ $P_{t}(y, k)=\lambda_{k}^{t} \exp (i k y)$ which requires

$$
\lambda_{k}=\exp (i k)+\exp (-i k), \quad k \in[0, \pi] .
$$

We now exploit the degeneracy of $\lambda_{k}$ by noting that $P_{t}(y, k)=\lambda_{k}^{t} \exp (i k y)$, and $P_{t}(y,-k)$ satisfy (2.8) so we try a more general form of solution, $R_{t}(y, k)=\lambda_{k}^{t}(A(k) \exp (i k y)+$ $B(k) \exp (-i k y))$ for $\bar{Z}_{t}^{\mathcal{S}}\left(y^{i} \rightarrow y\right)$. If this is substituted into (2.9), then we must have $A / B=\exp \left(-i \theta_{k}\right)$, where

$$
\exp \left(i \theta_{k}\right)=-\frac{\lambda_{k}-\kappa \exp (-i k)}{\lambda_{k}-\kappa \exp (i k)} .
$$

Thus, the most general form of the solution for $\bar{Z}_{t}^{S}\left(y^{i} \rightarrow y\right)$ satisfying (2.8) and (2.9) is

$$
W_{t}(y)=\int_{0}^{\pi} A(k) \lambda_{k}^{t}\left(\exp (i k y)+\exp \left(-i k y+i \theta_{k}\right)\right) d k .
$$

It remains to choose the arbitrary function $A(k)$ such that the initial condition (2.10) is satisfied. If we choose $A(k)=\left(\exp \left(-i k y^{i}\right)+\exp \left(i k y^{i}-i \theta_{k}\right)\right) / 2 \pi$, then by a few changes of variable, we can rewrite our trial solution as

$$
\begin{aligned}
\bar{Z}_{t}^{S}\left(y^{i} \rightarrow y^{f}\right) & =\frac{1}{2 \pi} \int_{-\pi}^{\pi} \lambda_{k}^{t}\left[\exp \left(i k\left\{y^{f}-y^{i}\right\}\right)+\exp \left(i k\left\{y^{f}+y^{i}\right\}+i \theta_{k}\right)\right] d k \\
& =\oint_{|u|=1}(u+1 / u)^{t}\left(u^{y^{f}-y^{i}}+S(u) u^{y^{f}+y^{i}}\right) \frac{d u}{u},
\end{aligned}
$$

where

$$
S(u)=-\frac{u+\frac{1}{u}-\frac{\kappa}{u}}{u+\frac{1}{u}-\kappa u} .
$$


If we Taylor expand the denominator of the integrand (uniformly convergent for $\kappa$ contained in any closed subinterval of $(0,2))$ and integrate term by term using $\oint_{|u|=1} u^{M} d u / u$ $=2 \pi i \delta_{M, 0}$, with $M$ as an integer, it shows that the result is equivalent to evaluating the "constant term" of a formal Laurent power series. Thus, (2.15) is equivalent to

$$
\bar{Z}_{t}^{S}\left(y^{i} \rightarrow y^{f}\right)=\mathrm{CT}\left[\left(z+\frac{1}{z}\right)^{t}\left(z^{y^{f}-y^{i}}+S(z) z^{y^{f}+y^{i}}\right)\right],
$$

where $z$ is considered a formal variable. More precisely, we form the quotient field, $F_{R((z))}$, from a ring, $R((z))$, of formal Laurent power series, $\sum_{n \geq n_{0}} a_{n} z^{n}$, where $n_{0}$ is an integer and $a_{n}$ are elements of some integral domain. We then define the constant term only on the subring, $F_{R((z))}^{*}$, of $F_{R((z))}$ isomorphic to $R((z))$. Thus, if $\gamma=\sum_{n \geq n_{0}} a_{n} z^{n} \in$ $F_{R((z))}^{*}$, then

$$
\mathrm{CT}[\gamma]=a_{0}
$$

Note that this restricts the denominator series of $F_{R((z))}^{*}$ to the form $\sum_{n \geq n_{0}} a_{n} z^{n}$ with $a_{n_{0}}=1$. Clearly, this is the case in (2.17). It is then straightforward to show that an integral $\oint_{|u|=1} f(u) d u / u$ of a rational function $f(u)$ is equal to $\mathrm{CT}[f(z)]$, with $f(z)$ considered as an element of $F_{R((z))}^{*}$ if and only if $f(u)$, considered as a function, is analytic in a punctured disc centered on the origin of radius greater than 1.

Evaluating the constant term gives

$$
\begin{aligned}
\bar{Z}_{t}^{S}\left(y^{i} \rightarrow y^{f}\right)= & \left(\begin{array}{c}
t \\
\frac{1}{2}\left(t-y^{f}+y^{i}\right)
\end{array}\right)-\left(\begin{array}{c}
t \\
\frac{1}{2}\left(t-y^{f}-y^{i}-2\right)
\end{array}\right) \\
& +\sum_{n \geq 1}(\kappa-1)^{n}\left\{\left(\begin{array}{c}
t \\
\frac{1}{2}\left(t-2 n-y^{f}-y^{i}+2\right)
\end{array}\right)\right. \\
& \left.-\left(\begin{array}{c}
t \\
\frac{1}{2}\left(t-2 n-y^{f}-y^{i}-2\right)
\end{array}\right)\right\}
\end{aligned}
$$

Note that the integral (2.15) can also be evaluated by residues. Our aim is, however, to have a method of evaluating $N$-dimensional integrals; the residue method does not seem to generalize easily whilst the constant term method does [1].

If $t=0$ then, for $y^{i}, y^{f} \in \mathcal{S}_{\infty}$ the only case a non-zero result is obtained is if $y^{i}=y^{f}$ in which case (2.19) reduces to (2.10). Thus, the Ansatz (2.15) is justified.

\subsection{Many Non-Intersecting Paths in the Half Plane}

In this section, we will solve the one-wall, $N$ non-intersecting path problem using the method of partial difference equations.

The generating function (or partition function), $\bar{Z}_{t}^{\mathcal{N}}\left(\mathbf{y}^{i} \rightarrow \mathbf{y}\right)$, of configurations in which the paths start at $\mathbf{y}^{i} \in \stackrel{p}{\mathcal{U}}$ s satisfies the following partial difference equation:

$$
\bar{Z}_{t}^{\mathcal{N}}\left(\mathbf{y}^{i} \rightarrow \mathbf{y}\right)=\sum_{e_{1}= \pm 1} \ldots \sum_{e_{N}= \pm 1} \bar{Z}_{t-1}^{\mathcal{N}}\left(\mathbf{y}^{i} \rightarrow \mathbf{y}+\mathbf{e}\right), \quad y_{1}>0
$$


with the boundary condition

$$
\bar{Z}_{t}^{\mathcal{N}}\left(\mathbf{y}^{i} \rightarrow\left(0, y_{2}, \ldots, y_{n}\right)\right)=\kappa \sum_{e_{2}= \pm 1} \ldots \sum_{e_{N}= \pm 1} \bar{Z}_{t-1}^{\mathcal{N}}\left(\mathbf{y}^{i} \rightarrow\left(1, y_{2}+e_{2}, \ldots, y_{N}+e_{N}\right)\right)
$$

meeting condition

$$
\bar{Z}_{t}^{\mathcal{N}}\left(\mathbf{y}^{i} \rightarrow \mathbf{y}\right)=0, \quad \text { if } y_{j}=y_{j+1} \text { for any } j \geq 1,
$$

and initial condition

$$
\bar{Z}_{0}^{\mathcal{N}}\left(\mathbf{y}^{i} \rightarrow \mathbf{y}\right)=\delta_{\mathbf{y}^{i}, \mathbf{y}}\left(1+(\kappa-1) \delta_{y_{1}, 0}\right),
$$

where $\mathbf{e}=\left(e_{1}, \ldots e_{N}\right)$. Equation (2.21) allows for the wall (at $\left.y_{1}=0\right)$ at step $t$, where the only possibility for the previous step is $y_{1}=1$. This ensures that negative coordinates never arise. Equation (2.22) ensures the paths do not intersect. The variable $\kappa$ counts the number of sites the lowest path has in common with the wall.

Analogous to the one-path method, the first step is to separate the step variable $t$ and the height variables $\mathbf{y}$ in Equation (2.20). We then look for particular solutions to (2.20) and use a linear combination to solve (2.21) and (2.22). This will use up all but $N$ of the arbitrary constants. The final constants are then used to satisfy the initial condition, (2.23). Thus, for the first step we substitute $\Lambda_{\mathbf{k}}^{t} \exp (i \mathbf{k} \cdot \mathbf{y})$ into (2.20) for $\bar{Z}_{t}^{\mathcal{N}}\left(\mathbf{y}^{i} \rightarrow \mathbf{y}\right)$, where $\mathbf{k}=\left(k_{1}, \ldots, k_{N}\right)$. This substitution will satisfy Equation (2.20) provided that

$$
\Lambda_{\mathbf{k}}=\prod_{\alpha=1}^{N} \lambda_{k_{\alpha}}, \quad \lambda_{k_{\alpha}}=\exp \left(i k_{\alpha}\right)+\exp \left(-i k_{\alpha}\right), \quad k_{\alpha} \in[0, \pi] .
$$

If no two of the $k_{\alpha}$ are equal, then any permutation of the $k_{\alpha}$ will also give a linearly independent solution of (2.20) with the same value of $\Lambda_{\mathbf{k}}$. Furthermore, if the sign of any of the $k_{\alpha}$ is reversed, another linearly independent solution is obtained with the same value of $\Lambda_{\mathbf{k}}$. Taken together any fixed set of $k_{\alpha}$ with $k_{\alpha} \in[0, \pi]$, no two of which are equal, gives us $2^{N} N$ ! solutions. We find below that by considering all possible subsets of $\left\{k_{1}, k_{2}, \ldots, k_{N}\right\}$, we have sufficient solutions to satisfy the initial and boundary conditions and hence do not require further solutions. Specifically, let $P_{N}$ be the set of $N$ ! permutations of $\{1,2, \ldots, N\}, \sigma=\left(\sigma_{1}, \sigma_{2}, \ldots, \sigma_{N}\right) \in P_{N}$, and $\eta=\left(\eta_{1}, \ldots, \eta_{N}\right)$, where

$\eta_{\alpha}= \pm 1$, then $A \frac{\eta}{\sigma} \prod_{\alpha=1}^{N} \exp \left(i \eta_{\alpha} k_{\alpha} y_{\sigma_{\alpha}}\right)$ is a solution of (2.20). The second step is to take a linear combination of the above solutions in order to satisfy the boundary and meeting conditions. The specific combination is inspired by the Bethe Ansatz for the eigenvectors of the six-vertex model [10]. The explanation for the success of this guess is given in Section 3. Thus, we try

$$
\bar{Z}_{t}^{\mathcal{N}}=\sum_{\sigma \in P_{N}} \sum_{\eta_{1}= \pm 1} \cdots \sum_{\eta_{N}= \pm 1} \int_{0}^{\pi} d \mathbf{k} A \frac{\eta}{\sigma} \Lambda_{\mathbf{k}}^{t} \prod_{\alpha=1}^{N} \exp \left(i \eta_{\alpha} k_{\alpha} y_{\sigma_{\alpha}}\right) .
$$

If we assume the constants factorize

$$
A \frac{\eta}{\sigma}=A_{\sigma} \prod_{\alpha=1}^{N} B_{\alpha}^{\eta_{\alpha}}
$$


then with the following choice of factors

$$
\begin{aligned}
A_{\sigma} & =\varepsilon_{\sigma}, \\
\frac{B_{\alpha}^{+}}{B_{\alpha}^{-}} & =\exp \left(i \theta_{k_{\alpha}}\right),
\end{aligned}
$$

where $\varepsilon_{\sigma}$ is the signature of the permutation $\sigma$, the Ansatz satisfies (2.21) and (2.22). Since (2.28) only fixes the ratio of the constants, it leaves $N$ remaining constants. Thus, for the third step, we use the remaining constants to try and satisfy the initial condition (2.23). We try

$$
B_{\alpha}^{+}=\exp \left(-i k_{\alpha} y_{\alpha}^{i}\right)+\exp \left(i \theta_{k_{\alpha}}+i k_{\alpha} y_{\alpha}^{i}\right) .
$$

Substituting this into (2.25) and rearranging gives,

$$
\bar{Z}_{t}^{\mathcal{N}}\left(\mathbf{y}^{i} \rightarrow \mathbf{y}^{f}\right)=\sum_{\sigma \in P_{N}} \varepsilon_{\sigma} \int_{0}^{\pi} d \mathbf{k} \psi_{\mathbf{k}}^{*}\left(\mathbf{y}^{i}\right) \Lambda_{\mathbf{k}}^{t} \psi_{\mathbf{k}}\left(\mathbf{y}_{\sigma}^{f}\right)
$$

where $\mathbf{y}_{\sigma}^{f}=\left(y_{\sigma_{1}}^{f}, \ldots, y_{\sigma_{N}}^{f}\right)$,

$$
\psi_{\mathbf{k}}(\mathbf{y})=\prod_{\alpha=1}^{N}\left(\exp \left(i k_{\alpha} y_{\alpha}\right)+\exp \left(-i \theta_{k_{\alpha}}-i k_{\alpha} y_{\alpha}\right)\right)
$$

and $\psi_{\mathbf{k}}^{*}\left(\mathbf{y}^{i}\right)$ is the conjugate of $\psi_{\mathbf{k}}\left(\mathbf{y}^{i}\right)$. The multiple integral factorizes into a product of single integrals of the form

$$
\int_{-\pi}^{\pi} d k \lambda_{k}^{t}\left(\exp \left(i k\left(y-y^{\prime}\right)\right)+\exp \left(i \theta_{k}+i k\left(y+y^{\prime}\right)\right)\right),
$$

which corresponds to the $N=1$ case (i.e., the single path equation (2.15)). Hence, Equation (2.30) becomes

$$
\bar{Z}_{t}^{\mathcal{N}}\left(\mathbf{y}^{i} \rightarrow \mathbf{y}^{f}\right)=\sum_{\sigma \in P_{N}} \varepsilon_{\sigma} \prod_{\alpha=1}^{N} \bar{Z}_{t}^{\mathcal{S}}\left(y_{\alpha}^{i} \rightarrow y_{\sigma_{\alpha}}^{f}\right)
$$

which is the expansion of a determinant [2,3]. If $t=0, \bar{Z}_{0}^{\mathcal{N}}\left(\mathbf{y}^{i} \rightarrow \mathbf{y}^{f}\right)=\delta_{\mathbf{y}^{i}, \mathbf{y}^{f}}(1+$ $\left.(\kappa-1) \delta_{y_{1}^{i}, 0}\right)$ which agrees with (2.23) for $\mathbf{y}^{i}, \mathbf{y}^{f} \in \mathcal{U}_{\infty}$, thus justifying the Ansatz (2.29).

Remark. The factorization of the multiple integral (2.30) allows us to express the multiple integral as a product of single integrals or, equivalently, as the product of constant term expressions. Going back one step thus gives us

$$
\bar{Z}_{t}^{\mathcal{N}}\left(\mathbf{y}^{i} \rightarrow \mathbf{y}^{f}\right)=\mathrm{CT}\left[\sum_{\sigma \in P_{N}} \varepsilon_{\sigma} \prod_{\alpha=1}^{N}\left\{z_{\alpha}^{y_{\alpha}^{i}}+S\left(\frac{1}{z_{\alpha}}\right) z^{-y_{\alpha}^{i}}\right\}\left\{z_{\alpha}^{-y_{\alpha}^{f}}+S\left(z_{\alpha}\right) z^{y_{\alpha}^{f}}\right\}\right] .
$$

Although little is gained for non-intersecting walks by rewriting (2.30) this way, it is very significant in more difficult problems where the multiple integral does not factorize. In this case, the evaluation of the multiple integral is a major hurdle unless it can be written as a constant term of some formal series (in $N$ variables). This is precisely what happens in the case of osculating lattice paths [1]. 


\section{Relationship Between the Partial Difference Equation Method and the Transfer Matrix}

Having solved the $N$-path problem in the half plane via partial difference equations we now show there is an intimate relation between this method and that of diagonalizing transfer matrices via the Bethe Ansatz. As we will show, the relation manifests itself in two ways: (1) through the difference equations, the coefficients of which turn out to be matrix elements of an infinite-dimensional transfer matrix, and (2) in solving the difference equations by separating the step variable " $t$ " and the height variables, one ends up with another set of equations, the solution of which is equivalent to solving an infinite-dimensional eigenvector problem. Thus, the partial difference equations can be thought of as a method of representing the "no strip" (and hence, infinite-dimensional) transfer matrix method.

Let us consider a general $N$-path problem defined via the following set of partial difference equations. Now, the paths may be intersecting (but not cross), or not, and we place arbitrary weights on edges of the lattice that are occupied. Our only restriction is that, if there are $N$ occupied sites at $t=0$ then each column of edges has $N$ occupied steps. The generating function $\bar{Z}_{t}^{\mathcal{N}}\left(\mathbf{y}^{i} \rightarrow \mathbf{y}^{f}\right)$ of $N$ paths of length $t$ in the half plane is given by a set of partial difference equations:

$$
\bar{Z}_{t}^{\mathcal{N}}\left(\mathbf{y}^{i} \rightarrow \mathbf{y}^{f}\right)=\sum_{\mathbf{y} \in \mathcal{U}_{\infty}} \bar{Z}_{t-1}^{\mathcal{N}}\left(\mathbf{y}^{i} \rightarrow \mathbf{y}\right) \prod_{j=1}^{N} w\left(y_{j}, y_{j}^{f}\right), \quad \text { for } \quad \mathbf{y}^{i}, \mathbf{y}^{f} \in \mathcal{U}_{\infty} .
$$

Note that even though the paths are intersecting, we can always order the edges. Together with the initial condition

$$
\bar{Z}_{0}^{\mathcal{N}}\left(\mathbf{y}^{i} \rightarrow \mathbf{y}^{f}\right)=\delta_{\mathbf{y}^{i}, \mathbf{y}^{f}} V\left(\mathbf{y}^{i}\right),
$$

where

$$
V\left(\mathbf{y}^{i}\right)=\prod_{\alpha=1}^{N} v\left(y_{\alpha}^{i}\right)
$$

Equation (3.5) determines $\bar{Z}_{t}^{\mathcal{N}}\left(\mathbf{y}^{i} \rightarrow \mathbf{y}^{f}\right)$. Let us define the elements of a matrix $\left(\mathbf{R}_{N}\right)_{\mathbf{y}, \mathbf{y}}{ }^{f}$ as the coefficients of the partial difference equation:

$$
\left(\mathbf{R}_{N}\right)_{\mathbf{y}, \mathbf{y}^{f}}=\prod_{j=1}^{N} w\left(y_{j}, y_{j}^{f}\right)
$$

Now, the generating function $\overline{\bar{Z}}_{t}^{\mathcal{N}}\left(\mathbf{y}^{i} \rightarrow \mathbf{y}^{f}\right)$ of $N$ paths of length $t$ in a strip is related to $\overline{\bar{Z}}_{t-1}^{\mathcal{N}}\left(\mathbf{y}^{i} \rightarrow \mathbf{y}\right)$, by one of two one-step transfer matrices defined above depending on whether $\mathbf{y}^{f} \in \stackrel{e}{\mathcal{U}}_{L}$ or not, thus

$$
\overline{\bar{Z}}_{t}^{\mathcal{N}}\left(\mathbf{y}^{i} \rightarrow \mathbf{y}^{f}\right)= \begin{cases}\sum_{\mathbf{y} \in \stackrel{\mathcal{U}}{L}_{L}} \overline{\bar{Z}}_{t-1}^{\mathcal{N}}\left(\mathbf{y}^{i} \rightarrow \mathbf{y}\right) \stackrel{e o}{\left.\mathbf{T}_{N}\right)_{\mathbf{y}, \mathbf{y}},} & \text { for } \mathbf{y}^{f} \in \stackrel{o}{\mathcal{U}_{L}}, \\ \sum_{\mathbf{y} \in \stackrel{o}{\mathcal{U}}_{L}} \overline{\bar{Z}}_{t-1}^{\mathcal{N}}\left(\mathbf{y}^{i} \rightarrow \mathbf{y}\right)\left(\stackrel{o e}{\mathbf{T}_{N}}\right)_{\mathbf{y}, \mathbf{y}}, & \text { for } \mathbf{y}^{f} \in \stackrel{e}{\mathcal{U}_{L}}\end{cases}
$$


Now, so long as we do not consider paths that touch the upper wall, the elements of the matrices $\stackrel{e o}{\mathbf{T}_{N}}$ and $\stackrel{o e}{\mathbf{T}_{N}}$ are the same as those of $\left(\mathbf{R}_{N}\right)$ :

$$
\left(\stackrel{o e}{\mathbf{T}_{N}}\right)_{\mathbf{y}, \mathbf{y}^{\prime}}=\left(\mathbf{R}_{N}\right)_{\mathbf{y}, \mathbf{y}^{\prime}} \quad \mathbf{y} \in \stackrel{o}{\mathcal{U}_{L}} \text { and } \mathbf{y}^{\prime} \in \stackrel{e}{\mathcal{U}_{L}} \text { given } y_{N}, y_{N}^{\prime} \neq L
$$

and

$$
\left(\stackrel{e o}{\mathbf{T}_{N}}\right)_{\mathbf{y}^{\prime}, \mathbf{y}}=\left(\mathbf{R}_{N}\right)_{\mathbf{y}, \mathbf{y}^{\prime}} \quad \mathbf{y}^{\prime} \in \stackrel{e}{\mathfrak{U}_{L}} \text { and } \mathbf{y} \in \stackrel{o}{\mathcal{U}_{L}} \text { given } y_{N}, y_{N}^{\prime} \neq L
$$

This demonstrates that the coefficients of the partial difference equations are nothing other than the transfer matrix elements with a parity complication due to the lattice structure. The symmetry of the transfer matrix is partly determined by the choice of matrix element labeling which in turn is affected by the structure of the lattice. In our square lattice problem, only even or only odd heights are possible in any column of sites and the opposite parity is only allowed for adjacent columns.

Let us return to the problems solved in the previous section where we considered non-intersecting paths with the weights as described by Equation (2.6). Solving the partial difference equations (2.20-2.23) by separation of variables $\bar{Z}_{t}^{\mathcal{X}}\left(\mathbf{y}^{i} \rightarrow \mathbf{y}\right) \rightarrow \Lambda^{t} P(\mathbf{y})$ gives a set of equations

$$
\Lambda P\left(\mathbf{y}^{i} \rightarrow \mathbf{y}\right)=\sum_{\mathbf{y}^{\prime} \in \mathcal{U}_{L}} P\left(\mathbf{y}^{i} \rightarrow \mathbf{y}^{\prime}\right)\left(\mathbf{R}_{N}\right)_{\mathbf{y}^{\prime}, \mathbf{y}} .
$$

Solving these equations is the same as solving an infinite-dimensional eigenvector problem $\mathbf{R}|P\rangle=\Lambda|P\rangle$ with $\langle\mathbf{y} \mid P\rangle=P\left(\mathbf{y}^{i} \rightarrow \mathbf{y}\right)$ with $\mathbf{y} \in \mathcal{U}_{\infty}$. This explains our choice of the Bethe Ansatz-like trial solution for $P\left(\mathbf{y}^{i} \rightarrow \mathbf{y}\right)$ as this is precisely the type of Ansatz that is required in the diagonalization of the associated transfer matrices [2]. Note that the route to the Bethe Ansatz in both the transfer matrix and partial difference methods is to find solutions to the "bulk" equation such as (2.20) and then find linear combinations that satisfy the boundary and meeting conditions.

\section{Conclusions}

The partial difference equation method for solving several non-intersecting lattice path problems has been reviewed and extended. It has been shown to be well suited to the semi-infinite (though also fully infinite by default) scenario by providing a quick and easy method of solution. However, the method currently lacks the rigor of the transfer matrix method (when this is applied in a strip). We have demonstrated that the partial difference equation method is essentially the same as considering an infinitedimensional transfer matrix directly. Importantly, this method has led to the expression of the solution as a constant term of a formal Laurent series. The constant term formulation is crucial in intersecting path problems.

Acknowledgments. Financial support from the Australian Research Council is gratefully acknowledged by RB and ALO. JWE is grateful for financial support from the Australian Research Council and for the kind hospitality provided by the University of Melbourne during which time this research took place. 


\section{References}

1. R. Brak, Osculating lattice paths and alternating sign matrices, In: Proceedings of the "Formal Power Series and Algebraic Combinatorics" Conference, 1997.

2. R. Brak, J. Essam, and A.L. Owczarek, Exact solution of $N$ directed non-intersecting walks interacting with one or two boundaries, J. Phys. A., 1998, submitted.

3. R. Brak, J. Essam, and A.L. Owczarek, From the Bethe Ansatz to the Gessel-Viennot theorem, Ann. Combin., submitted.

4. R. Brak, J. Essam, and A.L. Owczarek, New results for directed vesicles and chains near an attractive wall, J. Stat. Phys. 93 (1998) 155-192.

5. M.E. Fisher, Walks, walls, wetting and melting, J. Stat. Phys. 34 (1984) 667-729.

6. P.J. Forrester, Probability of survival for vicious walkers near a cliff, J. Phys. A. 22 (1989) L609-L613.

7. I.M. Gessel and X. Viennot, Determinants, paths, and plane partitions, 1989, preprint.

8. I.M. Gessel and X. Viennot, Binomial determinants, paths, and hook length formulae, Advances in Math. 58 (1985) 300-321.

9. A.J. Guttmann, A.L. Owczarek, and X.G. Viennot, Vicious walkers and young tableaux i: Without walls, J. Phys. A. 31 (1998) 8123-8135.

10. E.H. Lieb, Residual entropy of square ice, Phys. Rev. 162 (1967) 162-172.

11. F.Y. Wu, Remarks on the modified potassium dihydrogen phosphate model of a ferroelectric, Phys. Rev. 168 (1968) 539-543. 